OPEN ACCESS

Edited by:

Feng Liu,

Tianjin Medical University General Hospital, China

Reviewed by:

Chuanjun Zhuo,

Tianjin Anding Hospital, China

Peipeng Liang,

Capital Normal University, China

${ }^{*}$ Correspondence:

Zhi Yang

yangz@smhc.org.cn

Yong Xu

xuyongsmu@vip.163.com

${ }^{t}$ These authors have contributed equally to this work

Specialty section:

This article was submitted to Brain Imaging Methods, a section of the journal

Frontiers in Neuroscience

Received: 02 July 2018 Accepted: 03 October 2018 Published: 22 October 2018

Citation:

Wang J, Hu Y, Li H, Ge L, Li J,

Cheng $L$, Yang Z, Zuo $X$ and $X u Y$

(2018) Connecting Openness and the Resting-State Brain Network:

A Discover-Validate Approach.

Front. Neurosci. 12:762.

doi: 10.3389/fnins.2018.00762

\section{Connecting Openness and the Resting-State Brain Network: A Discover-Validate Approach}

\author{
Junjie Wang ${ }^{1 \dagger}$, Yang $\mathrm{Hu}^{2 \dagger}$, Hong $\mathrm{Li}^{3 \dagger}$, Ling $\mathrm{Ge}^{4}$, Jing $\mathrm{Li}^{1}$, Long Cheng ${ }^{1}$, Zhi Yang ${ }^{2 *}$, \\ Xinian $\mathrm{ZuO}^{3}$ and Yong $\mathrm{Xu}{ }^{1,5,6 *}$ \\ ' Department of Psychiatry, First Hospital/First Clinical Medical College of Shanxi Medical University, Taiyuan, China, \\ ${ }^{2}$ Laboratory of Psychological Health and Imaging, Shanghai Mental Health Center, Shanghai Jiao Tong University Medical \\ School, Shanghai, China, ${ }^{3}$ Institute of Psychology, Chinese Academy of Sciences, Beijing, China, ${ }^{4}$ Department of Medical \\ Psychology, Shanxi Medical College of Continuing Education, Taiyuan, China, ${ }^{5}$ Multi-Disciplinary Team (MDT) Center \\ for Cognitive Impairment and Sleep Disorders, First Hospital of Shanxi Medical University, Taiyuan, China, ${ }^{6}$ Key Laboratory \\ of Cell Physiology in Shanxi Province, Taiyuan, China
}

In personality neuroscience, the openness-brain association has been a topic of interest. Previous studies usually started from difference in openness trait and used it to infer brain functional activity characteristics, but no study has used a "brain-first" research strategy to explore that association based on more objective brain imaging data. In this study, we used a fully data-driven approach to discover and validate the association between openness and the resting-state brain network. We collected data of 120 subjects as a discovery sample and 56 subjects as a validation sample. The Neuroticism Extraversion Openness Five-Factor Inventory (NEO-FFI) was used to measure the personality characteristics of all the subjects. Using an exploratory approach based on independent component analysis of resting-state functional magnetic resonance imaging (fMRl) data, we identified a parietal network that consisted of the precuneus and inferior parietal lobe. The inter-subject similarity of the parietal memory network exhibited significant associations with openness trait, and this association was validated using the 56-subject independent sample. This finding connects the openness trait to the characteristics of a neural network and helps to understand the underlying biology of the openness trait.

Keywords: openness, parietal memory network, resting-state fMRI, data-driven, functional connectome

\section{INTRODUCTION}

On the basis that human experiences and behaviors are generated by biological processes, primarily within the brain, we seem to assume that the regularities in these experiences and behaviors that constitute personality are associated with regularities in the biological functions of the brain, making personality neuroscience possible (Canli, 2008; DeYoung and Gray, 2009). There have been a large body of studies aiming to test and refine neurobiological theories of personality by using neuroscientific methods such as molecular genetics (Malhotra et al., 1996; Kalbitzer et al., 2009), electroencepholography (EEG; Diethelm and Simons, 1945; Stough et al., 2001; Schmidtke and Heller, 2004), and positron emission tomography (PET; Johnson et al., 1999; O'Gorman et al., 2006). As a major non-invasive brain mapping technique, functional magnetic resonance 
imaging (fMRI) has relatively high spatial and temporal resolution. In recent years, the number of researchers using fMRI to explore personality neuroscience increased.

Constituted of five broad domains: Extraversion, Neuroticism, Agreeableness, Conscientiousness, and Openness, the Five Factor Model (FFM) is the most widely used descriptive taxonomy of personality and provides a common language for personality research (Costa and McCrae, 1992; John et al., 2008). There have been numerous studies that linking the individual variation in the structure (Omura et al., 2005; Rauch et al., 2005; Xu and Potenza, 2012) and function (Adelstein et al., 2011; Passamonti et al., 2015; Xiang et al., 2016) of different brain regions to taxonomies of FFM. We were particularly interested in the personality trait openness, which is a normally distributed personality trait reflecting the tendency to engage in imaginative, creative, and abstract cognitive processes. As one of the most important dimensions of personality traits, openness has always been one of the research hotspots of personality-brain association.

Previous studies of openness-brain associations usually started from the personality trait (openness) concept that is formed by observer-dependent life experience and consensus. It is doubtful whether the concept of personality, which is not completely independent of the subjective experience of observers, can fully and objectively reflect the activity characteristics of brain function networks. Besides, the subjective concept-based group has the assumption that all subjects in one group is homogeneous in brain mechanism, i.e., they share common intrinsic connectivity networks (ICNs). In fact, previous studies have found that personality traits were mostly associated with brain functional connections that were inconsistently present across participants (Di Martino et al., 2009; Adelstein et al., 2011).

In the current study, we adopted a data-driven approach, generalized ranking and averaging independent component analysis by reproducibility (gRAICAR; Yang et al., 2008; Yang et al., 2012, 2014a; Tian et al., 2018), to investigate whether the subjects can be grouped into communities according to the characteristics of their ICNs. The openness scores were not used to define subject groups prior to gRAICAR analysis, but they were associated to the ICN-derived subject communities to interpret the findings in neuroimaging data. With the effort, we attempted to identify association between ICNs and openness, aiming to generate objective and reliable metric of openness based on brain's intrinsic functional activity characteristics.

We recruited 120 participants to investigate their characteristics of ICNs and associated the openness scores to the ICN-derived subject communities (Figure 1 shows a graphical demonstration of data analysis process). Furthermore, an independent sample of 56 subjects was recruited as a validation sample aiming to verify the findings from the discovery sample.

\section{MATERIALS AND METHODS}

\section{Participants}

A total of 176 participants, including 120 participants as a discovery sample (age range: 19-60 years; 53 males, years of education: $14.49 \pm 2.8$, see Table 1 for details) and 56 participants as a validation sample (age range: $18-26$ years; 12 males, years of education: $14.00 \pm 2.5$, see Table 1 for details) were recruited from Shanxi medical university or local communities. The inclusion criteria for the healthy participants were as follows: (1) age range from 18 to 60; (2) no serious physical diseases, pregnancy, or substance abuse; (3) no psychoactive substance use for at least 1 month; and (4) no history of mental disorder. The exclusion criteria for healthy subjects were as follows: (1) meet the criteria for any mental disorder according to DSM-IV; (2) family history of mental disorder; (3) history of taking antipsychotic drugs; and (4) unsuitability for MRI scans (metal implants or claustrophobia). The Ethics Committee at Shanxi Medical University approved the study protocol. Written informed consent was obtained from each participant prior to data acquisition. After the subjects completed the personality inventory and MRI scan, they subsequently received payment for their time. The methods were carried out in accordance with the approved guidelines.

\section{Behavior Data Acquisition}

We used NEO-FFI (Neuroticism Extraversion Openness FiveFactor Inventory) to measure personality characteristics of all participants. NEO-FFI has been proven to be consistent with the full version, and it also has good convergent validity with other personality inventories (Costa and McCrae, 1989; Parker and Stumpf, 1998; Kurtz and Sherker, 2003).

\section{MRI Data Acquisition}

All imaging data were collected using a 3.0 Tesla Siemens Trio MRI scanner at the Shanxi Provincial People's Hospital. Resting-state scans were acquired with an echo-planar imaging (EPI) sequence (32 axial interleaved slices, acquired from inferior to superior, $\mathrm{TR} / \mathrm{TE}=2,500 / 30 \mathrm{~ms}, \mathrm{FA}=90^{\circ}$, FOV $=240 \mathrm{~mm}$, matrix $=64 \times 64$, thickness $=4.0 \mathrm{~mm}$, gap $=0.0 \mathrm{~mm}, 212$ volumes, duration $\left.8^{\prime} 50^{\prime \prime}\right)$. High-resolution anatomical scans were acquired with a T1-weighted 3D MPRAGE sequence $\left(\mathrm{TR} / \mathrm{TE} / \mathrm{TI}=2,300 / 2.95 / 900 \mathrm{~ms}, \mathrm{FA}=9^{\circ}\right.$, slices $=160$, thickness $=1.2 \mathrm{~mm}, \mathrm{FOV}=225 \mathrm{~mm} \times 240 \mathrm{~mm}$, matrix $=240 \times 256)$.

\section{Image Preprocessing and Quality Control}

Both anatomical and resting-state fMRI images were performed using the Connectome Computation System (Zuo et al., 2013), an integrated data preprocessing system that connects AFNI, FSL, and Freesurfer (Cox, 2012; Fischl, 2012; Jenkinson et al., 2012). Structural images were first cleaned by using a spatially adaptive non-local mean filter to remove noise (Zuo and Xing, 2011) and fed into FreeSurfer 5.1 for extracting the brain as well as for segmenting the brain tissues into gray matter, white matter and cerebrospinal fluid. All images were converted into MNI152 space using Advanced Normalization Tools (ANTs; Avants and Gee, 2004).

The following preprocessing steps were applied to the resting-state fMRI images: (1) the first five volumes were discarded to allow MRI signal equilibration; (2) slice timing differences were corrected; (3) the head movements were 

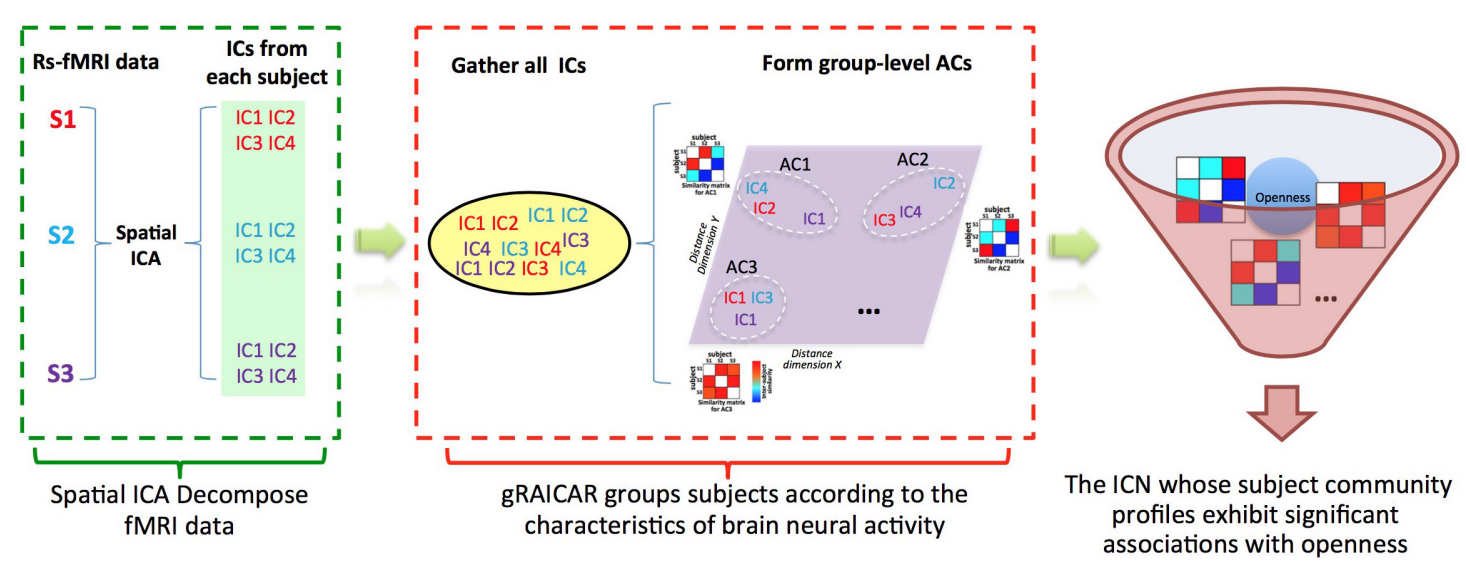

FIGURE 1 | Demonstration of data analysis workflow. For simplicity, we assume that there are only three subjects (denoted as S1, S2, and S3). First, the fMRI data of subjects are decomposed individually by using spatial independent component analysis (ICA) into spatial components (ICs). Assume that for each subject we can get four ICs that are color coded to indicate which subject they are from. The resultant ICs maps are presented in the green layer. Second, all of the ICs from individual subjects were pooled in gRAICAR (as presented in the yellow layer). We present a distance space depicting the similarity between all ICs in the purple layer. The intention of gRAICAR in this part is to identify ICs that are from different individuals but are close to each other (as marked with white dashed circles). The group-level aligned components (ACs) were formed by these clustered ICs sequentially, and a community detection algorithm can be applied to each AC to identify homogeneous subject communities among all subjects. Third, we try to seek the intrinsic connectivity networks whose subject community profiles exhibit significant associations with openness.

TABLE 1 | Descriptive statistics for the demographic characteristics of the two samples.

\begin{tabular}{lccccc}
\hline & Age range & Male & Female & Number & Percentage (\%) \\
\hline Sample (120) & $18-20$ & 0 & 2 & 2 & 1.67 \\
& $21-30$ & 17 & 16 & 33 & 27.50 \\
& $31-40$ & 10 & 10 & 20 & 16.67 \\
& $41-50$ & 16 & 28 & 44 & 36.66 \\
& $51-60$ & 10 & 11 & 21 & 17.50 \\
Sample (56) & $18-20$ & 3 & 21 & 24 & 42.86 \\
& $21-26$ & 9 & 23 & 32 & 57.14 \\
\hline
\end{tabular}

realigned over the entire scan; (4) the mean resting-state fMRI image was spatially normalized to MNI152 space via the combined registration of a rigid transformation of the individual structural images and nonlinear ANTs transformation; (5) the $4 \mathrm{D}$ data were standardized to a global mean intensity of 10,000 ; (6) the data were temporally band-pass $(0.01-0.1 \mathrm{~Hz})$ filtered.

The quality control procedure of anatomical images was performed by two researchers, including visual inspections of quality of tissue segmentation and head motion. For functional images, subjects would be excluded for excessive head motion evaluated using mean framewise displacement (meanFD), so that the meanFD was less than $0.2 \mathrm{~mm}$.

\section{gRAICAR Network Mining Analysis}

Generalized ranking and averaging independent component analysis by reproducibility was applied to the preprocessed functional images for the purpose of characterizing the intersubject similarity of the ICNs. By using gRAICAR, we could know how strong the one-to-one correspondence is, subsequently, we could reveal variations of brain maps across different subjects. Briefly, for each subject, the data were decomposed into spatial independent components (ICs) using the MELODIC module of FSL (Beckmann and Smith, 2004), and the spatial maps of the components were transformed into the MNI152 space. gRAICAR then pooled all of the ICs from each subject and matches them across all subjects to form a set of group-level aligned components (ACs). For each of the ACs, a spatial similarity matrix was computed to reflect the similarity between its comprising ICs, each representing a subject. This inter-subject similarity matrix represented a subject community profile that reflected potential subgroups of subjects sharing similar ICN characteristics.

To examine the significance of the associations between the inter-subject similarity derived from the ACs and the individual differences in the openness scores, we conducted a permutation test. First, each row of the inter-subject similarity matrix for each AC were summed to yield a degree of centrality for each subject in the given AC. The subjects were then classified into high openness score group (HOS, $n=83$ ) and low openness score group (LOS, $n=37$ ) according to the division standard of NEO-FFI. The between-group difference of the similarity degree was calculated. Under the null hypothesis that there was no significant difference in the similarity degree between the HOS and LOS subjects, the group identities of the subjects were then randomly permuted 8,000 times to yield a null distribution of the between-group difference of the similarity degrees. The percentile of the original betweengroup difference in the similarity degree indicated whether there was a significant association between the AC-derived inter-subject similarity and the individual difference in the openness classes. 


\section{RESULTS}

\section{Demographical and Behavioral Measures}

The demographical information and descriptive statistics for the NEO-FFI scores of all subjects in both experimental sample and validation sample are shown in Tables $\mathbf{1}$ and 2 . There was no significant difference between the two samples in terms of Openness scores $(t=-1.879, p=0.06)$, Extraversion scores $(t=1.027, p=0.30)$ and Agreeableness scores $(t=-1.424$, $p=0.15)$. There was significant difference between the two samples in terms of Neuroticism scores $(t=2.173, p=0.03)$ and Conscientiousness scores $(t=-3.728, p<0.01)$.

\section{gRAICAR Findings}

The gRAICAR algorithm identified thirty ACs. Based on previous literature (Beckmann et al., 2005; Damoiseaux et al., 2006, 2008) and their spatial patterns, $12 \mathrm{ACs}$ were found to represent functional ICNs. The remaining 18 components reflected artifacts like cerebrospinal fluid flow, physiological noise, and movement.

The inter-subject similarity matrix for each of the 12 ICNs reflected a subject community profile which reflect potential subgroups of subjects that share similar ICN characteristics. Thus, we explored the associations between the personality scores and the similarity matrix. We reordered the matrix according to hypothesized groupings and check whether the reorder the intersubject similarity matrix exhibits a clear cut-off between groups. By visual inspection, we found three ICNs that may be associated with three personality dimensions. However, after the analysis of voxel-wise functional connectivity strength, the Salience Network-Extroversion association $(t=-0.25, p=0.801)$ and the Sensorimotor Network-Neuroticism association $(t=1.43$, $p=0.156$ ) were not significant and therefore were excluded. In the following sections, we report remaining one ICN that were related to NEO-FFI-Openness classification.

\section{An ICN Associated With Openness Groups}

A parietal memory network (PMN, Gilmore et al., 2015) formed by the precuneus (PCU; $x=6 \mathrm{~mm}, y=-69 \mathrm{~mm}, z=60 \mathrm{~mm}$ in MNI space) and left and right inferior parietal lobule (1-IPL; $x=-39 \mathrm{~mm}, y=-84 \mathrm{~mm}, z=27 \mathrm{~mm}$ and r-IPL; $x=42 \mathrm{~mm}$, $y=-78 \mathrm{~mm}, z=33 \mathrm{~mm}$ ) reflected a subject community profile that was associated with the openness classification (Figure 2).

TABLE 2 | Descriptive statistics for the NEO-FFI scores of all participants in the two samples.

\begin{tabular}{lccc}
\hline $\begin{array}{l}\text { Dimension of } \\
\text { NEO-FFI }\end{array}$ & $\begin{array}{c}\text { Mean } \\
(\mathbf{1 2 0}) / \text { Mean (56) }\end{array}$ & $\begin{array}{c}\text { SD (120)/SD } \\
\mathbf{( 5 6 )}\end{array}$ & $\begin{array}{c}\text { Range } \\
\text { (120)/Range (56) }\end{array}$ \\
\hline Neuroticism & $31.93 / 34.14$ & $6.25 / 6.42$ & $16-39 / 21-49$ \\
Extraversion & $39.64 / 40.71$ & $6.67 / 5.96$ & $20-56 / 28-53$ \\
Openness & $38.90 / 37.79$ & $4.53 / 3.18$ & $28-53 / 30-43$ \\
Agreeableness & $42.00 / 41.13$ & $3.78 / 3.84$ & $31-51 / 34-49$ \\
Conscientiousness & $42.18 / 38.64$ & $6.05 / 5.45$ & $24-58 / 26-52$
\end{tabular}

The inter-subject similarity matrix of this network depicted similarities among all of the subjects.

After the permutation test, we found that there was a significant difference in the average similarity degree between the HOS subjects and the LOS subjects $(p<0.01)$. Visually, we found that the original inter-subject similarity matrix (Figure 2B) showed a clearer block when the subjects were reordered according the openness score (Figure 2C), with the block representing the subjects having highly similar ICN. These findings suggest that the PMN can distinguish the HOS subjects from the LOS subjects.

\section{Analysis of Voxel-Wise Functional Connectivity Strength}

The gRAICAR results revealed that the openness groups were associated with the inter-subject similarity reflected in the PMN. In order to provide supporting evidence for the gRAICAR findings from an independent methodology, we performed post hoc analyses to examine functional connectivity strength between the regions in the identified PMN and its associations with openness groups.

By applying a threshold of $Z>3.5$ and a cluster size $>30$ voxels to the brain map of the PMN, we obtained three regions of interests (ROIs), including the PCU, the l-IPL, and the r-IPL (Figure 3A). For each subject, the time series at each voxel within these ROIs was extracted to construct a voxel-wise correlation matrix. The correlation coefficients between voxels belonging to different ROIs were converted into Fisher's $Z$ values and then averaged to obtain a metric of inter-ROI functional connectivity for each connection. A network model highlighting the functional connectivity difference between the HOS and LOS subjects is shown in Figure 3B and the corresponding statistical comparisons are displayed in Figure 3C. One-way ANCOVA with age, sex, years of education, and meanFD as covariates was performed to examine the differences in inter-ROI functional connectivity between HOS and LOS subjects.

The results showed higher inter-ROI functional connectivity in HOS than in LOS subjects in connections between the 1-IPL and the r-IPL (connection 3 in Figure 3B, $F=6.216, p=0.014$ ).

\section{Independent Validation of the Findings}

In order to verify our findings in an independent sample, we used the same ROIs to conduct a functional connectivity analysis in the validation sample. Consist with our previous findings, the results revealed that functional connectivity strength between bilateral IPL in the HOS subjects is higher than that in the LOS subjects (connection 3 in Figure 3B, $F=5.421, p=0.024$ ) and the corresponding statistical comparisons are shown in Figure 3D.

\section{Correlations Between Connectivity Strength in PMN and Openness Scores}

In both the discovery and validation samples, openness scores significantly correlated with connectivity strength (Fisher's $Z$ ) between the 1-IPL and the r-IPL (Figure 4). We conducted a hierarchical regression analysis and the result indicated that the predictor of openness scores was connectivity strength between 
A

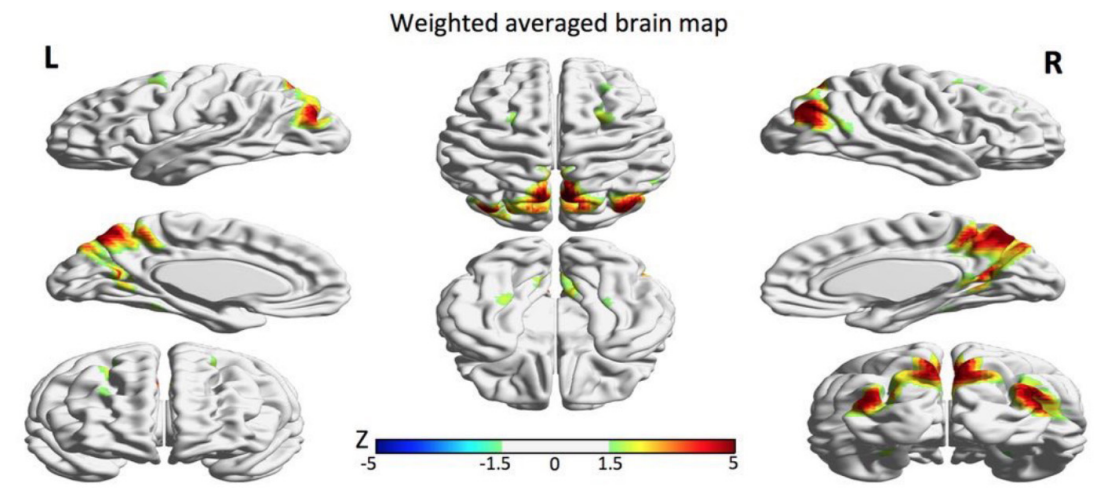

C Inter-subject similarity matrix

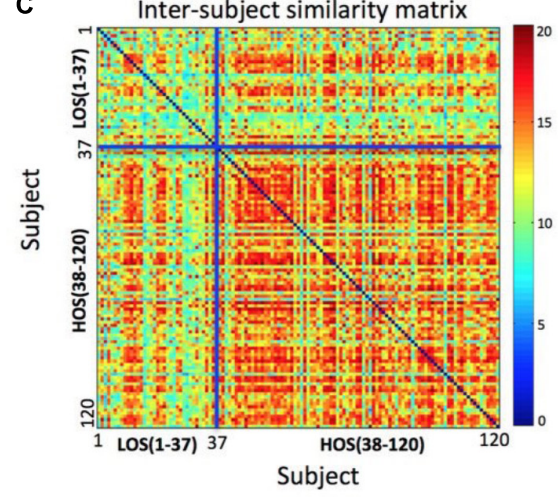

FIGURE 2 | The parietal memory network is associated with openness groups. (A) The parietal memory network rendered onto cortical surface of the brain. The network consists of three brain regions located in the precuneus, left inferior parietal lobule, and right inferior parietal lobule. The maps were thresholded at $|Z|>1.5$ for better visualization on the surfaces. (B) The similarity matrix of the parietal memory network across 120 subjects has no regular distribution. Both horizontal and vertical axes represent subjects. (C) Combined with the openness scores, the similarity matrix change into a regular distribution. Compared to the LOS subjects, the HOS subjects have a higher inter-subjects average similarity. For visualization purpose, the subjects are grouped into LOS and HOS groups, and the blue solid lines mark the boundary between the two groups. All surface maps are rendered in BrainNet Viewer (Xia et al., 2013).

the 1-IPL and the r-IPL. In the discovery sample, the predictor accounted for $7.8 \%$ of the model variance after controlling for age (adjusted $R^{2}=0.078, F=11.118, p<0.01$ ). In the validation sample, the predictor accounted for $6.9 \%$ of the model variance after controlling for age (adjusted $R^{2}=0.069, F=5.049, p<0.05$ ).

\section{DISCUSSION}

In this study, following a brain-first research strategy, we applied a discover-validate approach to investigate the characteristics of brain intrinsic networks with a sample of 120 healthy participants and found a reliable association between the PMN and NEOFFI-Openness, which was confirmed in a subsequent validation study with a sample of 56 participants. Specifically, the results showed higher inter-ROI functional connectivity in HOS than in LOS subjects in connections between the l-IPL and the r-IPL, and that people who have a higher functional integration of PMN exhibited a higher score on the NEO-FFI-Openness trait.

McCrae and Costa have pointed out that HOS people have a stronger information processing ability, so that the breadth and depth of their cognition has increased (McCrae and Costa, 1997).
Furthermore, in the FFM, openness is the only one trait that has a positive correlation with intelligence (Gray et al., 2003; DeYoung et al., 2005). As an important multimodal hubs in the brain for both structure and functional connectivity (Hagmann et al., 2008; Sporns, 2011; van den Heuvel and Sporns, 2011), PCU and IPL play important roles in the process of individual cognition (Kjaer et al., 2002; Lou et al., 2004; vogt and Laureys, 2005; Andrews-Hanna et al., 2010; Gilmore et al., 2015). Moreover, they have been closely related to the intelligence level of the individual (Owen et al., 2005; Jung and Haier, 2007). There has been a contention that openness may be related to the default mode network (DMN) functioning since both openness and the DMN are associated with imaginative cognition (DeYoung, 2014, 2015). Some evidence from personality neuroscience suggested that there is a stable positive correlation between openness and individual regions of the DMN (Raichle et al., 2001; Greicius et al., 2003; DeYoung et al., 2010; Adelstein et al., 2011; Beaty et al., 2016). The current study refined this argument to the PCU and IPL.

Changes in the PCU, which have been considered to play an essential role in individual consciousness (Cavanna, 2007), have previously been reported to be relevant to schizophrenia 
A
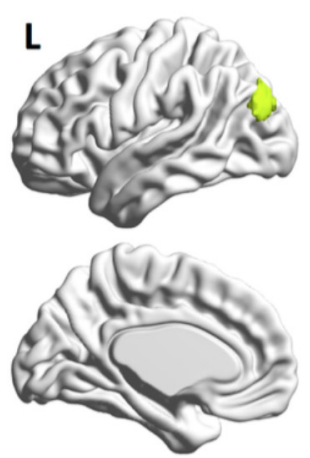

C

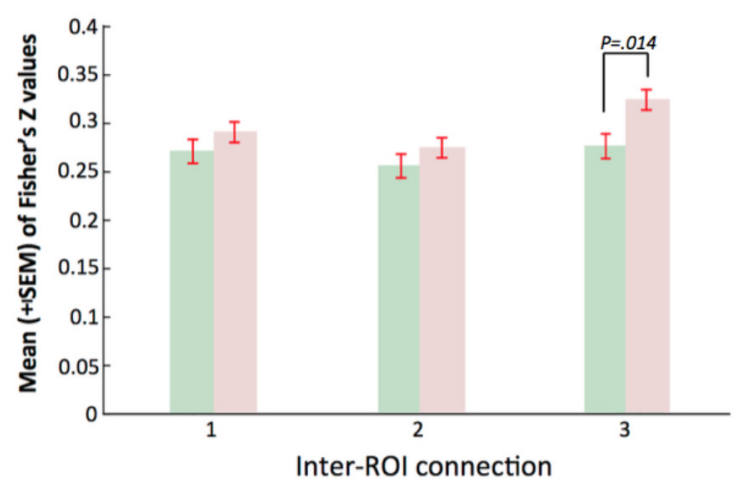

LOS
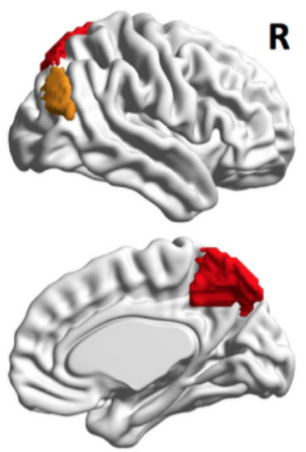

D

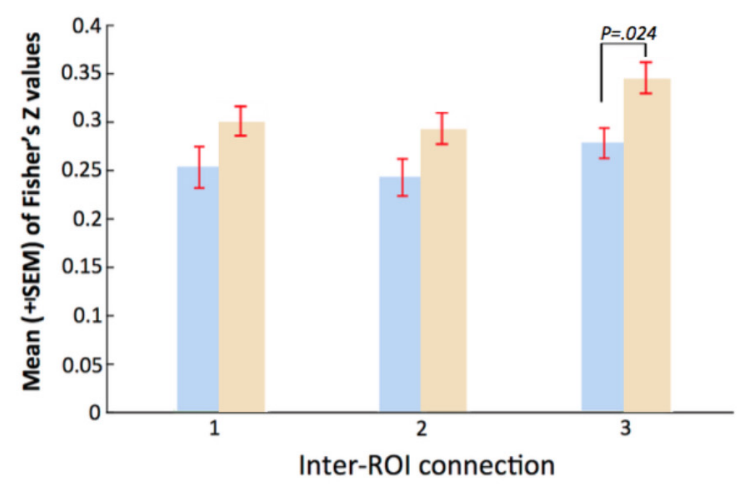

LOS

B

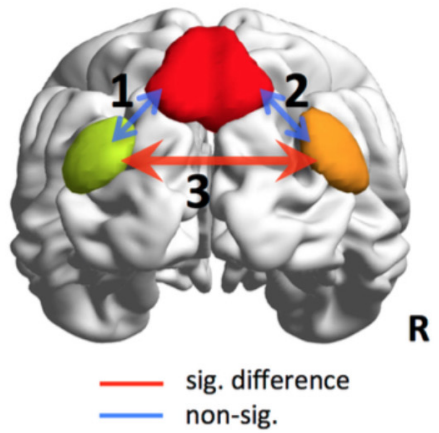

$\mathbf{R}$

HOS

FIGURE 3 | Voxel-wise functional connectivity analyses validate gRAICAR findings. (A) Three regions of interest including the PCU, the I-IPL and the r-IPL. (B) Map of the parietal memory network showing inter-regional connections exhibiting significant (red line) and non-significant (blue lines) differences in connectivity strength (Fisher's Z) between LOS and HOS groups. (C,D) Bar graphs showing statistical results in comparing functional connectivity strength between LOS and HOS groups. (C) presents the results from the discovery sample and (D) from validation sample. Labels along the horizontal axis correspond to the connections marked on (B). In both the discovery data and the validation samples, the connectivity between bilateral IPL shows significant difference between LOS and HOS groups.

A

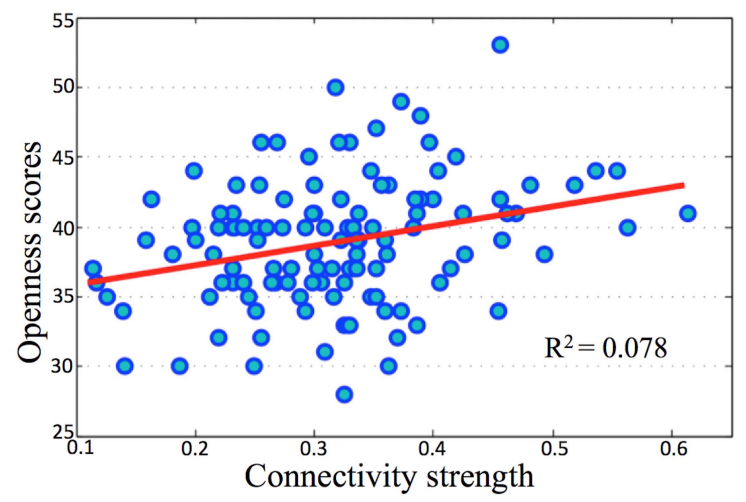

B

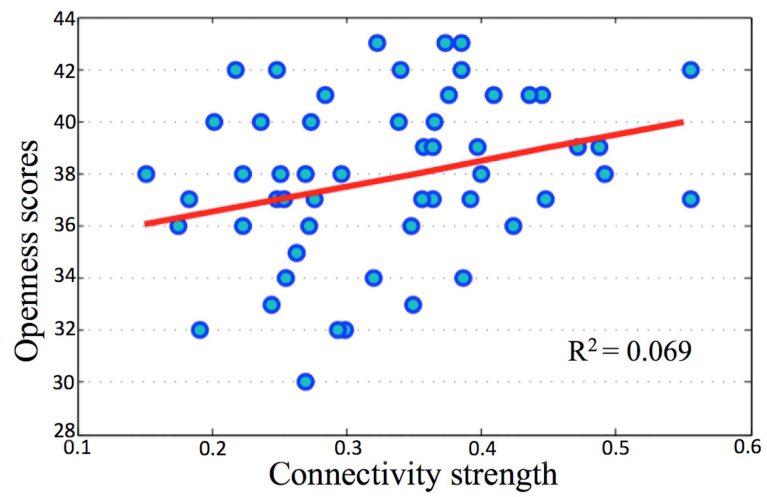

FIGURE 4 | Correlations between openness scores and connectivity strength in parietal memory network. The horizontal axes present connectivity strength (Fisher's $Z$ ) between the I-IPL and the r-IPL, and the vertical axes present the openness scores. (A) discovery sample, age and gender were controlled, $r=0.278, p<0.01$; (B) validation sample, age and gender were controlled, $r=0.277, p<0.05$.

(Buckner et al., 2008; Whitfield-Gabrieli et al., 2009; Kühn and Gallinat, 2013). One of our previous studies (Yang et al., 2014b) adopting the same neuroimaging data-mining approach used in the present study, gRAICAR, found that the PCU and bilateral angular gyri (AG, part of the IPL) were absent in earlyonset schizophrenia patients, and the results implied that the 
PCU-AG network is one of the primary targets affected by schizophrenia. Given the previous findings that patients with schizophrenia showed lower scores for openness compared with healthy subjects (Ohi et al., 2016), our findings that the PMN were statistically absent in LOS subjects can partly support the viewpoint that personality is one of important factors in the pathogenesis of schizophrenia since it affects subjects' cognition and social functioning as well as the patients' clinical symptoms (Ohi et al., 2016).

Yang et al. (2016) used a classical twin study design to examine the heritability of intrinsic functional network properties in 101 twin pairs, in which they showed that the activity of PMN is strongly heritable. Given our findings of a reliable association between the PMN and openness scores, it is reasonable to speculate a relationship between openness and genetics. In fact, twin studies have shown that personality traits are moderately heritable (Munafo et al., 2008) and have a relatively stable trajectory over time after early adulthood (Kupper et al., 2011). In addition, Amin et al. (2013) conducted a metaanalysis of four genome-wide linkage scans and identified 11q24 for openness to experience (NEO). By conducting a meta-analysis of NEO personality traits, de Moor et al. (2012) found significant associations for openness near the gene RASA1.

In the current study, we adopted a brain-first research strategy, trying to establish a hypothesis based on the differences of inter-individual brain intrinsic networks characteristics, and then inferred the personality trait (openness) associated with a certain brain intrinsic network characteristic. Psychologist has been observing the psychological world using categories derived from our own experiences, naming these categories using common sense words and searching for the counterparts of these categories within the brain. Since there has been a debate that these observer-dependent categories may not directly correspond (in a one-to-one fashion) to the observer-independent facts of the brain mechanism, thus, psychology may need a different set of psychological categories that more closely reflect the brain's activities in creating our mind and causing our behavior (Barrett, 2009).

Personality traits can be closely aligned with domains within the 'Research Domain Criteria' project (RDoC; Insel et al., 2010; Insel, 2014; Simmons and Quinn, 2014), which views psychiatric disorders as extremes of normal tendencies, and is intended to foster a biological analysis of behavior (Sanchez-Roige et al., 2018). While numerous fMRI studies aiming to establish the mental disorders classification system based on brain mechanism have examined psychiatric diseases (Fair et al., 2012, 2013; Yang et al., 2014b), relatively less work has been done on the brainfirst basis of RDoC traits such as personality. In addition, some new associations between brain networks and clinical

\section{REFERENCES}

Adelstein, J. S., Shehzad, Z., Mennes, M., Deyoung, C. G., Zuo, X. N., Kelly, C., et al. (2011). Personality is reflected in the brain's intrinsic functional architecture. PLoS One 6:e27633. doi: 10.1371/journal.pone.0027633 symptoms could only be detected in studies adopted RDoC strategy.

\section{LIMITATIONS AND FUTURE DIRECTIONS}

Combining findings of our research group in previous and the current work, it can be inferred that openness may be related to schizophrenia based on the characteristics of individual brain images. One of the prominent features of schizophrenia patients is impaired cognitive ability. Unfortunately, we did not have a systematic cognitive ability evaluations for our subjects. In future studies, relationships between ICNs, personality, and multi-dimensional cognitive abilities should be investigated.

As a study adopting the data-driven method, focusing only on differences in individual brain imaging features by fMRI is not comprehensive. The lower time resolution is a technically insurmountable defect. Future studies can collect higher timespatial resolution data by using EEG-fMRI. Genetic information, if conditions permit, should also be collect with the aim of establishing a more comprehensive research about biological basis of openness based on a gene-brain-behavior holistic perspective.

\section{AUTHOR CONTRIBUTIONS}

YX and ZY designed and supervised the study. JW, YH, and HL drafted the manuscript. LG, JL, and LC carried out the experimental procedures. $\mathrm{XZ}$ undertook the statistical analyses and reviewed the literature.

\section{FUNDING}

This study was supported by National Science Foundation of China (Grant Nos. 81571319, 81571756, 81501152, and 81671332), National Key Research and Development Program of China (2016YFC1307004), the Beijing Nova Program for Science and Technology (XXJH2015B079 to ZY), Ministry of Science and Technology of China (2016YFC1306800), Shanghai Municipal Education Commission-Gaofeng Clinical Medicine Grant Support (20171929), Hundred-talent Fund from Shanghai Municipal Commission of Health (2018BR17 to ZY), and Startup Fund from Shanghai Mental Health Center (13dz2260500, startup fund to ZY).

\section{ACKNOWLEDGMENTS}

We thank the participants of this study and the staff of the Shanghai Key Laboratory of Psychotic Disorders.

Amin, N., Hottenga, J. J., Hansell, N. K., Janssens, A. C., de Moor, M. H., Madden, P. A., et al. (2013). Refining genome-wide link- age intervals using a meta-analysis of genome-wide association studies identifies loci influencing personality dimensions. Eur. J. Hum. Genet. 21, 876-882. doi: 10.1038/ejhg. 2012.263 
Andrews-Hanna, J. R., Reidler, J. S., Huang, C., and Buckner, R. L. (2010). Evidence for the default network's role in spontaneous cognition. J. Neurophysiol. 104, 322-335. doi: 10.1152/jn.00830.2009

Avants, B., and Gee, J. C. (2004). Geodesic estimation for large deformation anatomical shape averaging and interpolation. Neuroimage 23, 139-150. doi: 10.1016/j.neuroimage.2004.07.010

Barrett, L. F. (2009). The future of psychology: connecting mind to brain. Perspect. Psychol. Sci. 4, 326-339. doi: 10.1111/j.1745-6924.2009.01134.x

Beaty, R. E., Kaufman, S. B., Benedek, M., Jung, R. E., Kenett, Y. N., Jauk, E., et al. (2016). Personality and complex brain networks: the role of openness to experience in default network efficiency. Hum. Brain Mapp. 37, 773-779. doi: $10.1002 / \mathrm{hbm} .23065$

Beckmann, C. F., DeLuca, M., Devlin, J. T., and Smith, S. M. (2005). Investigations into resting-state connectivity using independent component analysis. Philos. Trans. R. Soc. Lond. B Biol. Sci. 360, 1001-1013. doi: 10.1098/rstb.2005.1634

Beckmann, C. F., and Smith, S. M. (2004). Probabilistic independent component analysis for functional magnetic resonance imaging. IEEE. Trans. Med. Imaging 23, 137-152. doi: 10.1109/TMI.2003.822821

Buckner, R. L., Andrews-Hanna, J. R., and Schacter, D. L. (2008). The brain's default network: anatomy, function, and relevance to disease. Ann. N. Y. Acad. Sci. 1124, 1-38. doi: 10.1196/annals.1440.011

Canli, T. (2008). “Toward a "molecular psychology" of personality," in Handbook of Personality: Theory and Research, eds O. P. John, R. W. Robins, and L. A. Pervin (New York, NY: Guilford Press), 311-327.

Cavanna, A. E. (2007). The precuneus and consciousness. CNS. Spectr. 12, 545-552. doi: $10.1017 /$ S1092852900021295

Costa, P. T., and McCrae, R. R. (1989). The NEO-PI/NEO-FFI Manual Supplement. Odessa, FL: Psychological Assessment Resources.

Costa, P. T., and McCrae, R. R. (1992). NEO-PI-R Professional Manual: Revised NEO Personality and NEO Five-Factor Inventory (NEO-FFI). Odessa, FL: Psychological Assessment Resources.

Cox, R. W. (2012). AFNI: what a long strange trip it's been. Neuroimage 62, 743-747. doi: 10.1016/j.neuroimage.2011.08.056

Damoiseaux, J. S., Beckmann, C. F., Arigita, E. J., Barkhof, F., Scheltens, P., Stam, C. J., et al. (2008). Reduced resting-state brain activity in the "default network" in normal aging. Cereb. Cortex 18, 1856-1864. doi: 10.1093/cercor/bhm207

Damoiseaux, J. S., Rombouts, S. A., Barkhof, F., Scheltens, P., Stam, C. J., Smith, S. M., et al. (2006). Consistent resting-state networks across healthy subjects. Proc. Natl. Acad. Sci. U.S.A. 103, 13848-13853. doi: 10.1073/pnas.0601417103

de Moor, M. H., Costa, P. T., Terracciano, A., Krueger, R. F., de Geus, E. J., et al. (2012). Meta-analysis of genome-wide association studies for personality. Mol. Psychiatry 17, 337-349. doi: 10.1038/mp.2010.128

DeYoung, C. G. (2014). “Openness/Intellect: A dimension of personality reflecting cognitive exploration," in APA Handbook of Personality and Social Psychology: Personality Processes and Individual Differences, Vol. 4, eds M. Mikulincer, P. R. Shaver, M. Lynne Cooper, and R. J. Larsen (Washington, DC: American Psychological Association), 369-399.

DeYoung, C. G. (2015). Cybernetic big five theory. J. Res. Pers. 56, 33-58. doi: 10.1016/j.jrp.2014.07.004

DeYoung, C. G., and Gray, J. R. (2009). "Personality neuroscience: explaining individual differences in affect, behavior, and cognition," in The Cambridge Handbook of Personality Psychology, eds P. J. Corr and G. Matthews (New York, NY: Cambridge University Press), 323-346.

DeYoung, C. G., Hirsh, J. B., Shane, M. S., Papademetris, X., Rajeevan, N., and Gray, J. R. (2010). Testing predictions from personality neuroscience. Brain structure and the big five. Psychol. Sci. 21, 820-828. doi: 10.1177/0956797610370159

DeYoung, C. G., Peterson, J. B., and Higgins, D. M. (2005). Sources of openness/intellect: cognitive and neuropsychological correlates of the fifth factor of personality. J. Pers. 73, 825-858. doi: 10.1111/j.14676494.2005.00330.x

Di Martino, A., Shehzad, Z., Kelly, C., Roy, A. K., Gee, D. G., Uddin, L. Q., et al. (2009). Relationship between cingulo-insular functional connectivity and autistic traits in neurotypical adults. Am. J. Psychiatry 166, 891-899. doi: 10. 1176/appi.ajp.2009.08121894

Diethelm, O., and Simons, D. J. (1945). Electroencephalographic findings in psychopathic personalities. J. Nerv. Ment. Dis. 102, 611-614.

Fair, D. A., Bathula, D., Nikolas, M. A., and Nigg, J. T. (2012). Distinct neuropsychological subgroups in typically developing youth inform heterogeneity in children with ADHD. Proc. Natl. Acad. Sci. U.S.A. 109, 6769-6774. doi: 10.1073/pnas.1115365109

Fair, D. A., Nigg, J. T., Iyer, S., Bathula, D., Mills, K. L., Dosenbach, N. U., et al. (2013). Distinct neural signatures detected for ADHD subtypes after controlling for micro-movements in resting state functional connectivity MRI data. Front. Syst. Neurosci. 6:80. doi: 10.3389/fnsys.2012.00080

Fischl, B. (2012). FreeSurfer. Neuroimage 62, 774-781. doi: 10.1016/j.neuroimage. 2012.01.021

Gilmore, A. W., Nelson, S. M., and McDermott, K. B. (2015). A parietal memory network revealed by multiple MRI methods. Trends Cogn. Sci. 19, 534-543. doi: 10.1016/j.tics.2015.07.004

Gray, J. R., Chabris, C. F., and Braver, T. S. (2003). Neural mechanisms of general fluid intelligence. Nat. Neurosci. 6, 316-322. doi: 10.1038/nn1014

Greicius, M. D., Krasnow, B., Reiss, A. L., and Menon, V. (2003). Functional connectivity in the resting brain: a network analysis of the default mode hypothesis. Proc. Natl. Acad. Sci. U.S.A. 100, 253-258. doi: 10.1073/pnas. 0135058100

Hagmann, P., Cammoun, L., Gigandet, X., Meuli, R., Honey, C. J., Wedeen, V. J., et al. (2008). Mapping the structural core of human cerebral cortex. PLoS Biol. 6:e159. doi: 10.1371/journal.pbio.0060159

Insel, T., Cuthbert, B., Garvey, M., Heinssen, R., Pine, D. S., Quinn, K., et al. (2010). Research domain criteria $(\mathrm{RDoC})$ : toward a new classification framework for research on mental disorders. Am. J. Psychiatry 167, 748-751. doi: 10.1176/appi. ajp.2010.09091379

Insel, T. R. (2014). The NIMH research domain criteria (RDoC) project: precision medicine for psychiatry. Am. J. Psychiatry 171, 395-397. doi: 10.1176/appi.ajp. 2014.14020138

Jenkinson, M., Beckmann, C. F., Behrens, T. E., Woolrich, M. W., and Smith, S. M. (2012). FSL. Neuroimage 62, 782-790. doi: 10.1016/j.neuroimage.2011. 09.015

John, O. P., Naumann, L. P., and Soto, C. J. (2008). "Paradigm shift to the integrative Big Five trait taxonomy: history, measurement, and conceptual issues," in Handbook of Personality: Theory and Research, eds O. P. John, R. W. Robins, and L. A. Pervin (New York, NY: Guilford Press), 114-158.

Johnson, D. L., Wiebe, J. S., Gold, S. M., Andreasen, N. C., Hichwa, R. D., Watkins, G. L., et al. (1999). Cerebral blood flow and personality: a positron emission tomography study. Am. J. Psychiatry 156, 252-257.

Jung, R. E., and Haier, R. J. (2007). The Parieto-Frontal Integration Theory (PFIT) of intelligence: converging neuroimaging evidence. Behav. Brain Sci. 30, 135-154; discussion 154-187. doi: 10.1017/S0140525X07001185

Kalbitzer, J., Frokjaer, V. G., Erritzoe, D., Svarer, C., Cumming, P., Nielsen, F. A., et al. (2009). The personality trait openness is related to cerebral 5-HTT levels. Neuroimage 45, 280-285. doi: 10.1016/j.neuroimage.2008.12.001

Kjaer, T. W., Nowak, M., and Lou, H. C. (2002). Reflective self-awareness and conscious states: PET evidence for a common midline parietofrontal core. Neuroimage 17, 1080-1086. doi: 10.1006/nimg.2002.1230

Kühn, S., and Gallinat, J. (2013). Resting-state brain activity in schizophrenia and major depression: a quantitative meta-analysis. Schizophr. Bull. 39, 358-365. doi: $10.1093 /$ schbul/sbr151

Kupper, N., Boomsma, D. I., de Geus, E. J. C., Denollet, J., and Willemsen, G. (2011). Nine-year stability of type D personality: contributions of genes and environment. Psychosom. Med. 73, 75-82. doi: 10.1097/PSY.0b013e318 1 fdce 54

Kurtz, J. E., and Sherker, J. L. (2003). Relationship quality, trait similarity, and selfother agreement on personality ratings in college roommates. J. Pers. 71, 21-48. doi: 10.1111/1467-6494.t01-1-00005

Lou, H. C., Luber, B., Crupain, M., Keenan, J. P., Nowak, M., Kjaer, T. W., et al. (2004). Parietal cortex and representation of the mental Self. Proc. Natl. Acad. Sci. U.S.A. 101, 6827-6832. doi: 10.1073/pnas.0400049101

Malhotra, A. K., Virkkunen, M., Rooney, W., Eggert, M., Linnoila, M., and Goldman, D. (1996). The association between the dopamine D4 receptor (D4DR) 16 amino acid repeat polymorphism and novelty seeking. Mol. Psychiatry 1, 388-391.

McCrae, R. R., and Costa, P. T. (1997). "Conceptions and correlates of openness to experience," in Handbook of Personality Psychology, eds R. Hogan, J. A. Johnson, and S. R. Briggs (Boston: Academic Press), 826.

MunafoÌ, M. R., Yalcin, B., Willis-Owen, S. A., and Flint, J. (2008). Association of the dopamine $\mathrm{D} 4$ receptor (DRD4) gene and approach-related personality 
traits: meta-analysis and new data. Biol. Psychiatry 63, 197-206. doi: 10.1016/j. biopsych.2007.04.006

O’Gorman, R. L., Kumari, V., Williams, S. C., Zelaya, F. O., Connor, S. E., Alsop, D. C., et al. (2006). Personality factors correlate with regional cerebral perfusion. Neuroimage 31, 489-495. doi: 10.1016/j.neuroimage.2005.12.048

Ohi, K., Shimada, T., Nitta, Y., Kihara, H., Okubo, H., Uehara, T., et al. (2016). The five-factor model personality traits in schizophrenia: a meta-analysis. Psychiatry Res. 240, 34-41. doi: 10.1016/j.psychres.2016.04.004

Omura, K., Todd Constable, R., and Canli, T. (2005). Amygdala gray matter concentration is associated with extraversion and neuroticism. Neuroreport 16 , 1905-1908. doi: 10.1097/01.wnr.0000186596.64458.76

Owen, A. M., McMillan, K. M., Laird, A. R., and Bullmore, E. (2005). N-back working memory paradigm: a meta-analysis of normative functional neuroimaging studies. Hum. Brain Mapp. 25, 46-59. doi: 10.1002/hbm.20131

Parker, W. D., and Stumpf, H. (1998). A validation of the five-factor model of personality in academically talented youth across observers and instruments. Pers. Individ. Differ. 25, 1005-1025. doi: 10.1016/S0191-8869(98) 00016-6

Passamonti, L., Terracciano, A., Riccelli, R., Donzuso, G., Cerasa, A., Vaccaro, M., et al. (2015). Increased functional connectivity within mesocortical networks in open people. Neuroimage 104, 301-309. doi: 10.1016/j.neuroimage.2014.09.017

Raichle, M. E., MacLeod, A. M., Snyder, A. Z., Powers, W. J., Gusnard, D. A., and Shulman, G. L. (2001). A default mode of brain function. Proc. Natl. Acad. Sci. U.S.A. 98, 676-682. doi: 10.1073/pnas.98.2.676

Rauch, S. L., Milad, M. R., Orr, S. P., Quinn, B. T., Fischl, B., and Pitman, R. K. (2005). Orbitofrontal thickness, retention of fear extinction, and extraversion. Neuroreport 16, 1909-1912. doi: 10.1097/01.wnr.0000186599.66243.50

Sanchez-Roige, S., Gray, J. C., MacKillop, J., Chen, C. H., and Palmer, A. A. (2018). The genetics of human personality. Genes Brain Behav. 17:e12439. doi: 10.1111/gbb.12439

Schmidtke, J. I., and Heller, W. (2004). Personality, affect and EEG: predicting patterns of regional brain activity related to extraversion and neuroticism. Pers. Individ. Differ. 36, 717-732. doi: 10.1016/S0191-8869(03)00129-6

Simmons, J. M., and Quinn, K. J. (2014). The NIMH Research Domain Criteria (RDoC) Project: implications for genetics research. Mamm. Genome 25, 23-31. doi: 10.1007/s00335-013-9476-9

Sporns, O. (2011). The human connectome: a complex network. Ann. N. Y. Acad. Sci. 1224, 109-125. doi: 10.1111/j.1749-6632.2010.05888.x

Stough, C., Donaldson, C., Scarlata, B., and Ciorciari, J. (2001). Psychophysiological correlates of the NEO PI-R openness, agreeableness and conscientiousness: preliminary results. Int. J. Psychophysiol. 41, 87-91. doi: 10.1016/S01678760(00)00176-8

Tian, F., Wang, J., Xu, C., Li, H., and Ma, X. (2018). Focusing on the differences of resting-state brain networks, using a data-driven approach to explore the functional neuroimaging characteristics of extraversion trait. Front. Neurosci. 12:109. doi: 10.3389/fnins.2018.00109

van den Heuvel, M. P., and Sporns, O. (2011). Rich-club organization of the human connectome. J. Neurosci. 31, 15775-15786. doi: 10.1523/JNEUROSCI.3539-11. 2011

vogt, B. A., and Laureys, S. (2005). Posterior cingulate, precuneal and retrosplenial cortices: cytology and components of the neural network correlates of consciousness. Prog. Brain Res. 150, 205-217. doi: 10.1016/S0079-6123(05) 50015-3
Whitfield-Gabrieli, S., Thermenos, H. W., Milanovic, S., Tsuang, M. T., Faraone, S. V., McCarley, R. W., et al. (2009). Hyperactivity and hyperconnectivity of the default network in schizophrenia and in first-degree relatives of persons with schizophrenia. Proc. Natl. Acad. Sci. U.S.A. 106, 1279-1284. doi: 10.1073/pnas. 0809141106

Xia, M., Wang, J., and He, Y. (2013). BrainNet Viewer: a network visualization tool for human brain connectomics. PLoS One 8:e68910. doi: 10.1371/journal.pone. 0068910

Xiang, Y., Kong, F., Wen, X., Wu, Q., and Mo, L. (2016). Neural correlates of envy: regional homogeneity of resting-state brain activity predicts dispositional envy. Neuroimage 142, 225-230. doi: 10.1016/j.neuroimage.2016. 08.003

Xu, J., and Potenza, M. N. (2012). White matter integrity and five-factor personality measures in healthy adults. Neuroimage 59, 800-807. doi: 10.1016/ j.neuroimage.2011.07.040

Yang, Z., Chang, C., Xu, T., Jiang, L., Handwerker, D. A., Castellanos, F. X., et al. (2014a). Connectivity trajectory across lifespan differentiates the precuneus from the default network. Neuroimage 89, 45-56. doi: 10.1016/j.neuroimage. 2013.10.039

Yang, Z., LaConte, S., Weng, X., and Hu, X. (2008). Ranking and averaging independent component analysis by reproducibility (RAICAR). Hum. Brain Mapp. 29, 711-725. doi: 10.1002/hbm.20432

Yang, Z., Xu, Y., Xu, T., Hoy, C. W., Handwerker, D. A., Chen, G., et al. (2014b). Brain network informed subject community detection in early-onset schizophrenia. Sci. Rep. 3:5549. doi: 10.1038/srep05549

Yang, Z., Zuo, X. N., McMahon, K. L., Craddock, R. C., Kelly, C., de Zubicaray, G. I., et al. (2016). Genetic and environmental contributions to functional connectivity architecture of the human brain. Cereb. Cortex 26, 2341-2352. doi: 10.1093/cercor/bhw027

Yang, Z., Zuo, X. N., Wang, P., Li, Z., LaConte, S. M., Bandettini, P. A., et al. (2012). Generalized RAICAR: discover homogeneous subject (sub)groups by reproducibility of their intrinsic connectivity networks. Neuroimage 63, 403414. doi: 10.1016/j.neuroimage.2012.06.060

Zuo, X. N., and Xing, X. X. (2011). Effects of non-local diffusion on structural MRI preprocessing and default network mapping: statistical comparisons with isotropic/anisotropic diffusion. PLoS One 6:e26703. doi: 10.1371/journal.pone. 0026703

Zuo, X. N., Xu, T., Jiang, L., Yang, Z., Cao, X. Y., He, Y., et al. (2013). Toward reliable characterization of functional homogeneity in the human brain: preprocessing, scan duration, imaging resolution and computational space. Neuroimage 65, 374-386. doi: 10.1016/j.neuroimage.2012.10.017

Conflict of Interest Statement: The authors declare that the research was conducted in the absence of any commercial or financial relationships that could be construed as a potential conflict of interest.

Copyright (c) 2018 Wang, Hu, Li, Ge, Li, Cheng, Yang, Zuo and Xu. This is an open-access article distributed under the terms of the Creative Commons Attribution License (CC BY). The use, distribution or reproduction in other forums is permitted, provided the original author(s) and the copyright owner(s) are credited and that the original publication in this journal is cited, in accordance with accepted academic practice. No use, distribution or reproduction is permitted which does not comply with these terms. 